\title{
ORIGINAL
}

\section{LA FORMACIÓN PRÁCTICA EN ENFERMERÍA EN LA ESCUELA UNIVERSITARIA DE ENFERMERÍA DE LA COMUNIDAD DE MADRID. OPINIÓN DE LOS ALUMNOS Y DE LOS PROFESIONALES ASISTENCIALES. UN ESTUDIO CUALITATIVO CON GRUPOS DE DISCUSIÓN}

\author{
Cristina Pérez Andrés (1), Almudena Alameda Cuesta (2) y Carmen Albéniz Lizarraga (3).
}

(1) Subdirección General de Promoción de la Salud y Epidemiología. Dirección General de Salud Pública. Ministerio de Sanidad y Consumo.

(2) Escuela Universitaria de enfermería de la Comunidad de Madrid. Leganés, Madrid.

(3) Consejería de Sanidad. Comunidad de Madrid.

\section{RESUMEN}

Fundamentos: En las escuelas de enfermería el contraste entre lo que se enseña en las aulas y lo que se practica en los centros asistenciales suele provocar en los alumnos una gran confusión. El objetivo de esta investigación es conocer la opinión de los estudiantes y de los profesionales de los centros asistenciales en los que realizan sus prácticas clínicas acerca de las mismas, para detectar sus problemas y ver si existen diferencias existen entre atención primaria y atención especializada.

Métodos: La investigación se realizó durante el primer semestre del año 2000 con metodología cualitativa, a través de cuatro grupos de discusión, formados por alumnos, exalumnos, tutores de atención primaria y profesionales de enfermería de los hospitales en los que se realizan las prácticas. La intervención inicial utilizada fue indirecta.

Resultados: El discurso de los estudiantes de enfermería y de sus tutores, sobre las prácticas durante la diplomatura muestra la insatisfacción de ambos colectivos. En todos los grupos aparece la angustia como protagonista de sus actividades tanto docentes como discentes y asistenciales. La falta de reconocimiento social condiciona su falta de identificación como colectivo, y ambos que la angustia esté presente. El reconocimiento de la licenciatura para sus estudios universitarios y el desarrollo de las especialidades contribuiría a su reconocimiento social y a su identificación como colectivo profesional.

Conclusiones: Mientras no se resuelva la angustia que los profesionales de enfermería tienen, y que trasladan a los alumnos durante la realización de las prácticas, no se podrá alcanzar mayor nivel de satisfacción con las mismas, ni por parte de los tutores ni por parte de los alumnos.

Palabras clave: Escuelas de enfermería. Estudiantes de enfermería. Práctica del docente de enfermería. Investigación en educación de enfermería. Investigación cualitativa. Personal de enfermería. Salud laboral en enfermería. Satisfacción profesional. Especialidades de enfermería. Ansiedad. Relación médicos-enfermeras. Reconocimiento social.

\section{Correspondencia:}

Almudena Alameda Cuesta

Escuela Universitaria de Enfermería de la Comunidad de Madrid. Avda Orellana, s/n. Recinto Hospital Severo Ochoa. Leganés. 28911 Madrid

Correo electrónico: e.u.enfermería@comadrid.es

\section{ABSTRACT}

The Opinion of Nursing Students and Professionals Concerning Nursing

Training in the Community of Madrid, Spain: A Discussion Groups Study

Background: In the nursing schools, the contrast between what is taught in the classrooms and what is practiced at the health care centers usually creates a great deal of confusion on the part of the students. The objective of this research is to ascertain the opinion of the students and of the professionals at the health care centers where they are doing their training with regard thereto in order to detect their problems and see what differences exist between primary and specialized care.

Methods: This research was conducted throughout the first half of 2000 employing qualitative methodology, by means of four discussion groups comprised of students, former students, primary care training advisors and nursing professionals at the hospitals where the students of the school in question are doing their nursing training. The initial involvement employed was indirect.

Results: The comments of the nursing students and of their training advisors with regard to the practice nursing during the diploma studies reveal dissatisfaction on the part of both of these groups. In all of the groups point out anxiety as the leading factor involved in their teaching as well as learning activities and during professional training. The lack of identification as a group of poffesionals seems to be related to the lack of recognition on the part of the others, the demand for a degree being granted for their college studies and for the setting up of specialties would contribute to their social recognition and, as a result thereof, to their identification as a professional group.

Conclusions: Until a solution is provided to the anxiety which the nursing professionals feel with regard to their professional practice, which they pass on to their students during nursing training, it will not be possible to achieve a higher degree of satisfaction with nursing training experiences either on the part of the training advisors or on the part of the students.

Keywords: Job satisfaction. Nurses. Nursing, Practical. Specialties, nursing. Schools, Nursing. Occupational Health nursing. Students, nursing. Qualitative research. Anxiety. Physician-Nurse Relations. Social Identification. 


\section{INTRODUCCIÓN}

La integración de la teoría y la práctica en la formación académica es una de las mayores preocupaciones de las instituciones docentes. Las escuelas de enfermería no son una excepción y el contraste entre lo que se enseña en las aulas y lo que se aprende en los centros asistenciales en los que se realizan las prácticas clínicas, suele sumir a los alumnos en una gran confusión. Sería demasiado pretencioso, incluso utópico, querer llegar a un nivel de concordancia perfecta entre la teoría y la práctica de una materia. Es el sujeto implicado en el proceso de aprendizaje el que utiliza los medios a su alcance para integrar teoría y práctica de una manera dinámica. A pesar de esta realidad, desde el punto de vista docente siempre hay que procurar que exista coherencia entre la formación teórica y la formación práctica, para evitar que aparezca lo que algunos autores han llamado «shock de realidad». Este vendría provocado por la disonancia entre cómo se cree que deberían ser las cosas y cómo son en realidad, y sus consecuencias principales son la intensa desorientación y la angustia del estudiante ${ }^{1}$.

Algunos autores han introducido conceptos como praxis o práctica reflexiva en el campo de la docencia en enfermería ${ }^{2}$. Estos términos nos hablan de una filosofía pedagógica y metodológica de la formación; en ella el eje central lo constituye la práctica. Pero ésta es mucho más que una mera aplicación instrumentalizada de lo que el estudiante aprende en el aula o que una repetición de lo observado durante los períodos que pasa en los centros asistenciales. Consiste en una visión mucho más amplia de la labor de enfermería, en la que cada problema es único, inmerso en una realidad determinada, y cuya resolución radica en la acción desde la reflexión. Desde este punto de vista, las prácticas clínicas toman gran relevancia y la necesidad de que el aprendizaje en este ámbito sea riguroso se convierte en algo irrenunciable.
Entre los profesionales de enfermería nos encontramos con tres sectores: el asistencial, el docente y el gestor ${ }^{3}$. Esta división, en lugar de suponer un enriquecimiento de todo el colectivo de enfermería, provoca más bien un estado de permanente conflicto, ya que cada uno de ellos proyecta una imagen distinta marcada por sus objetivos particulares, los cuales, en lo que se refiere a la formación de estudiantes, parecen ir en direcciones distintas. Los asistenciales piensan que los docentes no preparan a los alumnos para enfrentarse con la realidad; los docentes, a su vez, mantienen que los asistenciales no asumen su compromiso docente y que son reacios a abrirse a las nuevas tendencias que marcan el desempeño profesional de enfermería. Con esta situación es realmente muy difícil transmitir a los alumnos una imagen unificada y complementaria sobre la teoría y la práctica.

De los resultados obtenidos en un estudio realizado en la EUE Príncipes de España de Barcelona $^{4}$, durante los cursos académicos 1988-89 al 1991-92 se pudo deducir que el aumento en la calidad de la comunicación entre docentes y asistenciales había mejorado, a su vez, la percepción de los estudiantes sobre la consecución de sus objetivos en la práctica clínica.

Existen experiencias en varios países europeos, así como en Estados Unidos, sobre la aproximación teoría-práctica durante los estudios de enfermería $a^{5-7} y$, aunque hay diferencias significativas entre las diversas propuestas, en todas ellas se parte de una unión sólida entre las direcciones de las escuelas y las direcciones de enfermería de los centros asistenciales. Bajo esta única línea directiva, los profesionales de enfermería tendrían una triple responsabilidad: asistencial, docente e investigadora.

La Escuela de Enfermería de la Comunidad de Madrid comenzó a funcionar en 1991 dependiendo de la Consejería de Sanidad y de la Universidad Autónoma de Madrid. Su metodología docente se puede resumir en 
tres puntos fundamentales: sentido de la realidad, interrelación teoría-práctica y participación activa de los alumnos ${ }^{8}$. En este marco conceptual queda planteada la necesidad de integrar teoría y práctica y, aunque no es más que un comienzo, el hecho de enunciarla permite ver las dificultades de su aplicación y desarrollar estudios para evaluarla. En este ámbito se planteó la realización de esta investigación en la Escuela Universitaria de Enfermería de la Comunidad de Madrid.

El objetivo de esta investigación es conocer la opinión de los estudiantes de la Escuela Universitaria de Enfermería de la Comunidad de Madrid y de los profesionales de los centros asistenciales en los que realizan sus prácticas clínicas acerca de las mismas, para detectar sus problemas y ver qué diferencias existen entre atención primaria y atención especializada.

\section{SUJETOS Y MÉTODOS}

Las relaciones que se establecen en las actividades docentes de cualquier enseñanza en general y de la enfermería en particular, entre el profesorado y los estudiantes, conforma, a la hora de su investigación, un objeto complejo que incluye las relaciones personales y la intersubjetividad de las personas implicadas en el proceso docente y discente. La complejidad de la estructura de dicho objeto justifica el empleo de la metodología cualitativa o estructural en el proceso de su investigación. Lo fundamental de ésta es que trabaja con el análisis e interpretación del lenguaje (verbal o no verbal) obtenido en condiciones de investigación, siendo las técnicas más características la observación participante, las historias de vida, la entrevista abierta y el grupo de discusión ${ }^{9-12}$.

La investigación se realizó durante el primer semestre del año 2000 con metodología cualitativa, obteniéndose la información mediante grupos de discusión, por ser esta la técnica más adecuada para nuestro objeto de estudio y la más característica de las que se encuentran entre la metodología cualitati$\mathrm{va}^{13-16}$. Con el fin de permitir la expresión del discurso de los diferentes colectivos se diseñaron cuatro grupos para mantener la homogeneidad dentro de cada uno de ellos y la heterogeneidad entre grupos. En la tabla 1

Tabla 1

Características de cada grupo de discusión

\begin{tabular}{|c|c|c|c|c|c|c|c|}
\hline Grupo & tipo de participantes & $\begin{array}{l}\text { número de per- } \\
\text { sonas citadas }\end{array}$ & edad & sexo & contactador & $\begin{array}{c}\text { personas que } \\
\text { acudieron }\end{array}$ & Fecha reunión \\
\hline GD1 & Alumnos de $2 .^{\circ}$ y $3 .^{\circ}$ & 10 & $\begin{array}{l}\text { Menores de } \\
25 \text { años }\end{array}$ & $\begin{array}{l}\text { Se citó al menos } \\
\text { a dos varones }\end{array}$ & $\begin{array}{l}\text { Un alumno com- } \\
\text { pañero de los } \\
\text { participantes }\end{array}$ & 10 & $\begin{array}{l}17 \text { de enero de } 2000 \\
\text { de } 17: 00 \text { a } 18: 30\end{array}$ \\
\hline GD2 & $\begin{array}{l}\text { Exalumnos que hu- } \\
\text { bieran acabado sus } \\
\text { estudios en las dos } \\
\text { promociones anterio- } \\
\text { res a la fecha del es- } \\
\text { tudio }\end{array}$ & 10 & $\begin{array}{l}\text { Menores de } \\
25 \text { años }\end{array}$ & $\begin{array}{l}\text { Se citó al menos } \\
\text { a dos varones }\end{array}$ & $\begin{array}{l}\text { Un exalumno de } \\
\text { las dos últimas } \\
\text { promociones }\end{array}$ & 8 & $\begin{array}{l}24 \text { de enero de } 2000 \\
\text { de } 17: 00 \text { a } 18: 30\end{array}$ \\
\hline GD3 & $\begin{array}{c}\text { Profesionales de en- } \\
\text { fermería que traba- } \\
\text { jando en el hospital } \\
\text { tengan alumnos asig- } \\
\text { nados }\end{array}$ & 10 & $\begin{array}{c}\text { Entre } 25 \text { y } 50 \\
\text { años y con ex- } \\
\text { periencia labo- } \\
\text { ral mínima de } \\
\text { dos años }\end{array}$ & $\begin{array}{l}\text { Se citó al menos } \\
\text { a dos varones }\end{array}$ & $\begin{array}{c}\text { Un profesional } \\
\text { de enfermería } \\
\text { trabajador en un } \\
\text { hospital y siendo } \\
\text { docente }\end{array}$ & 3 & $\begin{array}{l}14 \text { de marzo de } 2000 \\
\text { de } 12: 00 \text { a } 13: 30\end{array}$ \\
\hline GD4 & $\begin{array}{c}\text { Profesionales de en- } \\
\text { fermería que traba- } \\
\text { jando en la atención } \\
\text { primaria sean tutores } \\
\text { de alumnos }\end{array}$ & 10 & $\begin{array}{l}\text { Entre } 25 \text { y } 50 \\
\text { años y con ex- } \\
\text { periencia míni- } \\
\text { ma como tuto- } \\
\text { res de un año }\end{array}$ & $\begin{array}{l}\text { Se citó al menos } \\
\text { a dos varones }\end{array}$ & $\begin{array}{l}\text { Un tutor de aten- } \\
\text { ción primaria }\end{array}$ & 10 & $\begin{array}{l}31 \text { de enero de } 2000 \\
\text { de } 17: 00 \text { a } 18: 30\end{array}$ \\
\hline
\end{tabular}


se refieren las características de las personas convocadas a cada grupo. Entre los alumnos no se incluyó a los de primer curso dado que cuando se realizó la investigación no habían comenzado a realizar prácticas clínicas.

A los contactadores se les facilitó una breve información sobre el estudio con el fin de que se la transmitieran a los componentes de los grupos, junto con la fecha, hora y lugar de la reunión. Los contactadores fueron retribuidos económicamente y los participantes en los grupos recibieron un regalo. Los grupos de discusión tuvieron lugar en la sala de reuniones de un Centro Cívico Municipal. La intervención inicial que se utilizó para provocar la producción de la información fue de tipo indirecto, ya que no explicitaba el objetivo de la investigación.

Durante la realización de los grupos una persona del equipo de investigación, ajena al ámbito de la Escuela, actuó como moderadora y otra como observadora. Las conversaciones se grabaron en cinta magnetofónica y posteriormente se transcribieron.

\section{RESULTADOS}

En la tabla 1 se puede observar cuántas personas acudieron a cada grupo, la fecha de realización de la reunión y el tiempo que duró cada una de ellas.

En cuanto al discurso de los grupos de discusión, se han encontrado estructuras semánticas comunes en todos ellos, que relacionan la profesión de enfermería y las prácticas clínicas, las cuales citamos a continuación.

\section{La angustia, el estrés, la ansiedad}

En primer lugar, en todos los grupos, independientemente de que estén compuestos por estudiantes o profesionales, ya sean de atención primaria o atención especializada, se cita el estrés, la angustia, la ansiedad y el miedo como principales protagonistas de su práctica (tabla 2).

\section{El trabajo en el hospital es muy estresante}

El discurso de todos los grupos manifiesta que el trabajo del hospital es muy estresante. La generalidad de los conocimientos adquiridos durante la diplomatura y la falta de definición de sus funciones no permiten desarrollar de forma protocolizada las actividades que realizan e impide su independencia de los médicos, así como la delimitación de roles con las auxiliares de enfermería. Se quejan de tener que realizar las funciones que ningún otro personal del hospital tienen señaladas pero que, sin embargo, tampoco son suyas. En los hospitales hay una disponibilidad excesiva para ser cambiados de un servicio a otro. En estos cambios no se tiene en cuenta ni la formación ni la experiencia a la hora de adscribirle a un servicio. También son motivo de estrés la sobrecarga de trabajo, por el número excesivo de enfermos a los que atiende cada profesional; el que las plantillas estén infradotadas, y el tener que doblar turnos. También el que el colectivo médico no tenga confianza en las decisiones tomadas por el personal de enfermería. Todas estas condiciones de trabajo les genera un estrés y un cansancio que puede justificar la falta de interés en objetivos comunes con el resto de sus compañeros (tabla 3 ).

\section{El trabajo en atención primaria es diferente}

Las diferencias del modo de trabajar entre atención primaria y atención especializada son tantas que plantean que puedan ser dos planes de estudio distintos o dos especialidades diferentes. Mientras que en el hospital echan de menos el trabajo en equipo, saben que en atención primaria sí lo hay, y también mejores relaciones con los médicos, funciones mejor definidas $\mathrm{y}$ menos carga asistencial. Pero en el grupo de 
Tabla 2

Verbatim relacionados con la angustia, el estrés, la ansiedad

Realmente depende de ti totalmente su vida, pero su cuidado, su bienestar, te entra mucha más angustia. Por eso a la hora de decir, jope, tengo que aprender a hacer muchas más cosas, porque es que depende de mí algo mucho más importante. (GD1)

Cuando empiezas a aprender, porque antes bueno, si es como estás es un como si estás aprendiendo, aprendiendo, pero es tal información que hay veces que no sabes ni por donde cogerla, se te amontona todo de golpe y dices y me tengo que acordar de todo esto... es una angustia. (GD1)

Es que en general luego la gente, cada uno dentro de la profesión es que hay muchísimas variantes, está el interino de los 10 años que está agobiado porque están saliendo las oposiciones, se tiene que sacar la plaza y además salen los traslados, lleva toda la vida en la pediatría, ahora resulta que le mandan a la medicina interna, que no ha estado con un adulto desde que terminó la carrera y ya no sabe si se va a poder amoldar; está la señora que está en el turno de tarde que quiere pasarse a la mañana que está todo el día luchando y cabreada y trata al paciente de determinada manera porque quiere estar en la mañana y está en la tarde y se lo ha quitado no sé quién, o sea es que es un continuo estrés o sea todo el mundo está, en general, está mal. (GD2)

Cuando llegan los suplentes les decimos no os preocupéis, no te preocupes porque sueñes por las noches y porque te vayas llorando a casa todos los días, que eso es lo habitual aquí. No debería ser así, pero todos los veranos, la gente se va llorando a casa. Es que es fuerte. Y tienes que decir, no te preocupes, no te preocupes que esto es así, le pasa a todo el mundo, no eres el único y es asi hasta que te adaptes y cojas mas práctica. Es triste, es que no salen preparados ni para el estrés ni para... (GD3)

Se hacen 4 cursos para quedar bien, para decir que hay una formación continuada, que son siempre los mismos cursos además, que para acceder a un curso a mi no me parece normal, pues yo me he apuntado a un curso, que es un curso para el estrés, que me parece que lo necesito hace tiempo, porque soy una persona muy estresada, pues me habré apuntado como siete veces y todavía no me ha tocado hacerlo, llevo 2 años apuntado en ese curso. (GD3)

- No nos hemos sentado y hemos dicho vamos a hacer una buena planificación, desde nunca y hemos ido cogiendo más y más y claro, llega un momento que tenemos overbooking, esto que dicen en los vuelos, igual, cuando ya tenemos overbooking pues resulta que es que no hacemos nada. Y dices mira, lo hacemos como salga y no nos planteamos si está bien o está mal.

- Lo que tenemos es burn out. Estamos quemados, claro.

- Estamos churruscados. (GD4)

Tabla 3

El trabajo en el hospital es muy estresante

Pero es que luego se cambia de una planta a otra. Yo estando en la UCI, ha habido gente que ha cambiado de servicio y llevaban 10 años trabajando ya y llegan alli y venía de urgencias a la UCI y era... la medicación se diluía totalmente distinta. Entonces la mujer años trabajando y y llegan alli y venía de urgencias a la UCI y era... la medicacion se diluia totalmente distinta. Entonces la mujer
llegaba los primeros días y estaba como nosotros con su cuadernillo, nada más que apuntando e ibas tú a preguntarle y te decia no, no, si a mi no me preguntes que... (GD1)

Yo tuve la experiencia de con los traslados de ahora, una enfermera que ha estado toda la vida trabajando con adultos, que pasan directamente a urgencias de pediatría, claro decía ¿qué es esto?, no, no tenía manejo, lo pasó fatal, hombre y imagino y lo seguirá pasando. (GD1)

Pues porque las condiciones de trabajo no son las mejores, hay veces que tienes que doblar, hay veces que tienes que triplicar, triplicar no, pero vamos, quedarte sin un fin de semana libre,... (GD2)

Y muchas veces entiendes, ¿no? cuando te decían, cuando estabas tú de prácticas, yo recuerdo estar de prácticas y decir, jo, es que yo he visto a la enfermera Fulanita de no sé qué, que es que va por la habitación y no hace no sé qué y no hace no sé cuantos y yo creo que es que eso hay que hacerlo. Jo, no, es que hay que estar viviendo cada día y la carga de trabajo que tiene cada enfermera ¿eh? (GD2)

Estás tan estresado, está muy bien todo eso, si yo lo sé, ojalá no se me olvide, porque algún día me gustaría practicarlo y hacerlo, pero hoy en día es imposible, es irrealizable que un enfermero tenga 16 enfermos, haga un proceso de atención de enfermería en condiciones, les dé apoyo emocional, esté con ellos, estudie, se reciclé, enseñe al alumno que tiene y haga investigación. (GD3)

Eres la persona que tiene que estar pendiente del trabajo del médico de que esté todo bien pautado, de que no haya ningún problema, vale; tienes que estar pendiente de las pruebas complementarias que se le piden y pegarte con rayos, con cocina, con laboratorio, con no sé qué, pegarte con todos los servicios del hospital. (GD3)

No y que luego cada enfermera, como se trabaja poco con protocolos, en el hospital cada enfermera va a su bola. (GD4) 
exalumnos, al referirse a la atención primaria, hablan de ella con diminutivos y expresiones que la catalogan en general como algo pequeño, lo que parece indicar cierto desprestigio de la misma entre ellos y, a pesar de las ventajas que sobre ella destacan, les gusta trabajar más en el hospital. De hecho todos los participantes de este grupo hacen suplencias en el hospital en el momento en el que se realiza esta investigación, ninguno en atención primaria.

Los tutores de atención primaria (GD4) piensan que trabajan de forma diferente al hospital, con más protocolos y, junto con la mayor confianza que tiene el médico en ellos, se sienten más autónomos. De hecho estos profesionales se plantean que la enfermería debería diseñar los planes de cuidados y dejar que las técnicas las haga otro personal, sugiriendo que ellos podrían tener un papel exclusivamente relacionado con la enfermería comunitaria y que deberían reducir su tiempo de consulta de enfermería. Así, plantean que la enfermería de atención primaria debería ser una especialidad. En la reforma de la atención primaria piensan que son ellos los que han asumido las funciones de la salud comunitaria, aunque todavía se sienten infrautilizados en este trabajo (tabla 4).

\section{Sobre la diplomatura, la licenciatura y la escuela}

El discurso de todos los grupos manifiesta que durante la diplomatura el estrés y la an-

Tabla 4

El trabajo en atención primaria es diferente

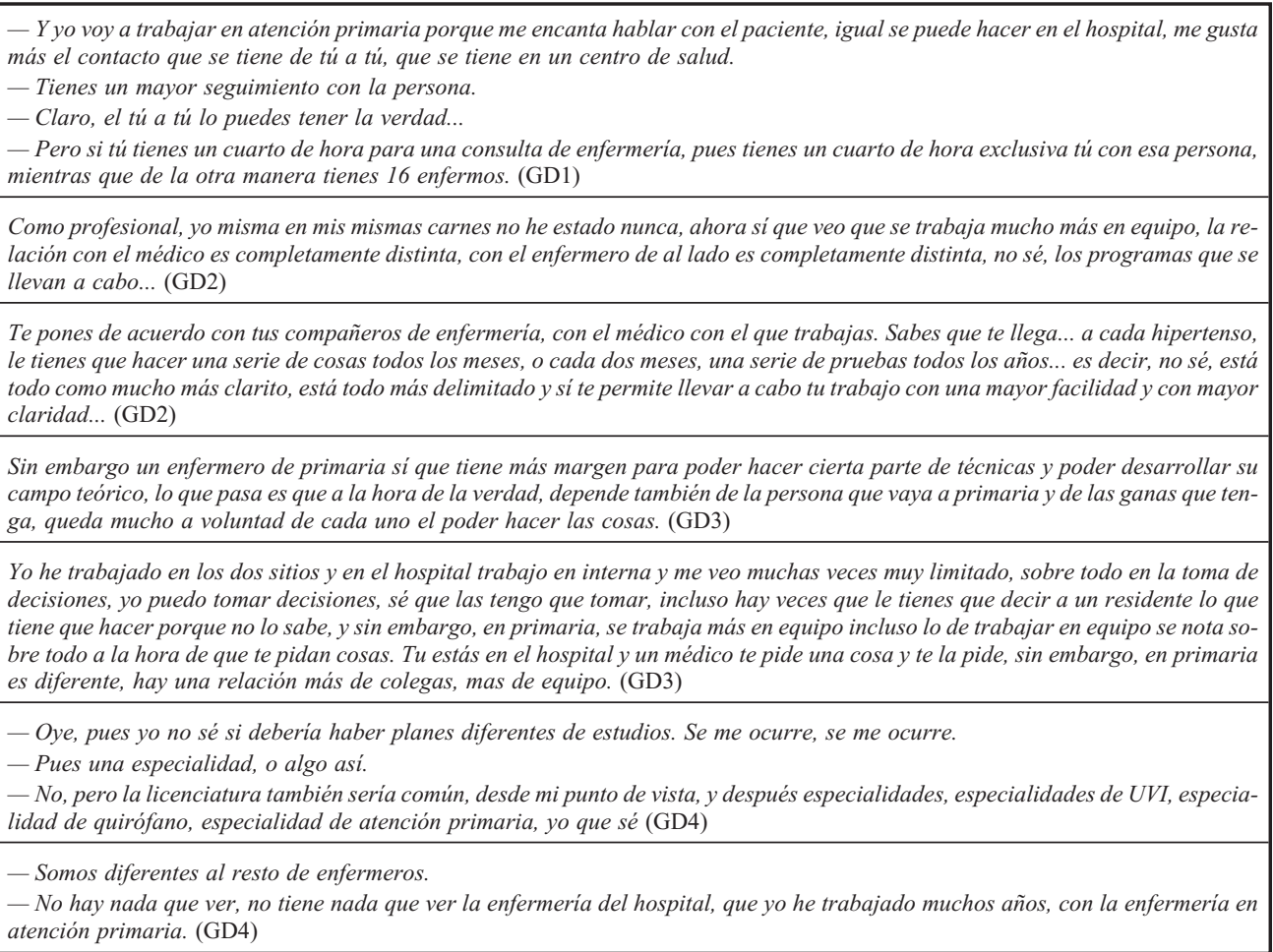


gustia se deben al excesivo trabajo que les supone la misma. Si hacen muchas prácticas les falta tiempo para las clases teóricas; si aumentan éstas, les falta tiempo para las prácticas, para los trabajos de grupo y para preparar los exámenes. A pesar de todo este trabajo, los estudiantes y los que ya están diplomados piensan que no salen suficientemente formados para trabajar, que sus conocimientos son excesivamente generales, y afirman que ambos problemas los resolverían a través de la licenciatura, que aumentaría la duración de la carrera, y con el desarrollo de las especialidades en enfermería.

Tanto los alumnos (GD1) como los exalumnos (GD2) opinan que la Escuela tiene un planteamiento muy exigente, que induce en ellos un espíritu muy competitivo. También, aunque valoran el trato personal con los profesores, critican la poca relación con la Universidad, de la que se sienten muy alejados, y el paternalismo de la Escuela. Aunque valo- ran las ventajas de estar en una institución con pocos alumnos, parece que pesan más los inconvenientes. Según ellos, la Escuela tiene un planteamiento demasiado orientado hacia atención primaria y la enfermería comunitaria, descuidando la formación en otras materias básicas, para lo que suelen poner de ejemplo la farmacología. Piensan que hay mucha diferencia entre los tutores de prácticas, tanto si son de atención primaria como especializada, y los profesores que trabajan en la Escuela, ya que éstos últimos sí están formados como docentes (tabla 5).

\section{Opinión que tienen de los profesionales de enfermería (Identidad como colectivo)}

Llama mucho la atención la mala opinión que los participantes en los grupos tienen sobre los profesionales de enfermería, a los que acusan de tener poco compañerismo, ser

Tabla 5

Sobre la diplomatura, la licenciatura y la escuela

Yo creo que es que realmente no es una carrera de tres años, que se reduce mucho menos por que las prácticas quitan un montón de tiempo que aunque tu puedas aprovecharlo para estudio, pero en lo que otras en lo que otras carreras tienen tres años y otras diplomaturas, ésta no tenemos tres años, te quitan un montón las prácticas y está todo mucho como muy concentrado. Yo creo que es como una licenciatura que la han metido en tres años y que debería ser mucho... yo creo que los mismos tres años alargados en una licenciatura. (GD1)

O también lo que yo pienso que igual que hay especialidades de matronas, especialidad de salud mental, urgencias darían para una especialidad, gerontología daría para enfermería gerontológica, daría para otra. Hacer, sí hacer un aparte, tener los tres años de tu carrera y aparte especialidades, que es cuando de verdad vas a empezar a profundizar y a conocer... a bueno estar especializada en un tema que aunque seas una enfermera por así decirlo como de base, de tener unos conocimientos amplios sobre todo, pero especializarte en algo, porque ahora mismo salimos y... como que sabemos muy poco. (GD1)

Creo que ya es entrar en cosas más profundas, creo que el planteamiento de esta carrera es hasta cierto punto bastante equivocado, hacemos una diplomatura y seguramente hacemos más horas que muchas licenciaturas. Nosotros salimos de casa durante los 3 años de carrera a las 7 de la mañana y hay días que se vuelve a las 11 de la noche, estás de prácticas pero estás haciendo una jornada laboral. Trabajas tus 7 horas, eres estudiante, vas a aprender, pero... (GD2)

De todas formas, si fuera más larga, si fuera una licenciatura la carrera, si que habría tiempo para toda la comunitaria y para toda la enfermería médica y para todo. (GD3)

Y posiblemente si se ampliara la licenciatura de enfermería el campo donde más se puede explorar es en ese, supongo. O el tema de las especialidades en enfermería, también es verdad que al final siempre valemos todos para todo. Porque sale una especialidad de matrona o de enfermería psiquiátrica y no sale uno de enfermeros de urgencias o de enfermeros de medicina interna o de... (GD3)

Pues por eso te digo, que tienes que hacer otra licenciatura de lo que sea, para poder impartir las clases de enfermería. A eso vamos, tienes que hacer antropología, psicología, sociología. (GD4)

Un aparejador tiene la misma titulación académica que nosotros y está cobrando medio millón de pesetas limpias al mes y es una carrera técnica exactamente igual que la nuestra y ya no es licenciatura, es un aparejador y como esos hay ejemplos múltiples. Te puedo traer nóminas. (GD4) 
pasivos e individualistas, ser poco trabajadores y estar poco interesados en los objetivos que debería tener la profesión. Ello ha originado que sus reivindicaciones se hayan quedado siempre sin realizar, por lo que en la actualidad continúan sin licenciatura, y lo que es peor, sin tener claro cuales son sus funciones ni, incluso, su profesión. En dos grupos, el de exalumnos y el de tutores de atención primaria, se relaciona la pasividad con el hecho de que el colectivo sea mayoritariamente femenino (tabla 6).

Tabla 6

Opinión que tienen de la profesión (Identidad como colectivo)

Es que sólo te falta trabajar en el campo de la enfermería, ese corporativismo que tienen los médicos en el campo de la enfermería no existe, y yo pienso que eso es en algo en lo que deberíamos trabajar. (GD1)

Hay mucha gente que te ve como futura competencia. Y más cuando... nosotros que estamos acabando hay muchos que te miran como si fueras la competencia de todos los días y más cuando no tienen la plaza fija. Te ven como que éste dentro de dos días me voy a tener que pelear con él para coger el puesto cuanto menos sepa y menos le cuente yo mejor, y te cuentan lo justo. (GD1)

Él lo que quiere es mantener su contrato y punto, y no le pidas que se movilice o que vaya a Dirección a poner una queja a su supervisora, porque vamos, no se le pasa por la cabeza. (GD2)

De todas formas yo creo que eso viene influido por la diferencia de formación que tienen unos enfermeros y otros, en cierta medida es culpa nuestra. Si tienes al lado un enfermero que estudió hace 30 años, y yo entiendo que es dificil reciclarse, pero que no se ha reciclado, pero evidentemente, cuando el médico llega y ve gente así pues es normal que no confie, yo tampoco lo haría. (GD3)

Y alguien que no lo está haciendo nunca, lo sabes y se lo dices y te dice que vale, que no lo va a hacer. Y entonces claro, dices, ¿para qué coño estoy aqui calentándome la cabeza, si además de hacer mi trabajo tengo que hacer lo que se ha dejado pendiente la persona que habia antes de mi? (GD3)

Yo digo que estamos muy cómodos y muy inmovilizados, no en el plan que tú has dicho, que sí que yo veo que nos hemos movido mucho, sino que, por ejemplo, a la hora de pedir sueldos nadie se ha movido, a la hora de pedir una licenciatura no nos hemos movido, a la hora de pedir que a los tutores nos reconozcan nuestro trabajo, no nos hemos movido, en este sentido digo cómodos. (GD4)

\section{Falta de reconocimiento social hacia su profesión}

En todos los grupos se manifiesta que la enfermería es una profesión muy poco valorada y poco reconocida por los pacientes, por sus amigos de otras carreras universitarias, por los médicos, por la sociedad en general y por ellos mismos. También se opina que aunque en la atención primaria existe más reconocimiento por parte de los médicos hacia su labor éste sigue sin darse por parte de la población.

Esta falta de reconocimiento social hacia la profesión parece dar origen al resto de unidades de sentido citadas anteriormente, dado que no permite la identidad del colectivo y genera la angustia que caracteriza su práctica profesional (tabla 7).

\section{Las prácticas durante la diplomatura}

El discurso tanto de los estudiantes de enfermería como de sus tutores, ya sean de atención primaria o de atención especializada, sobre las prácticas durante la diplomatura se encuentra atravesado por las estructuras semánticas identificadas anteriormente, mostrando la insatisfacción de ambos colectivos, alumnos y profesionales. La insatisfacción se argumenta con distintos factores más o menos objetivables en cada grupo.

En el caso de los estudiantes la relacionan con la falta de interés que perciben por parte de los tutores y la falta de formación de los mismos como docentes. La ansiedad que ello les crea se da tanto en las prácticas que realizan en atención primaria como en el 
Tabla 7

Falta de reconocimiento social hacia su profesión

No sólo enfermería, tú vas a un hospital y con eso de que los médicos siempre están por encima, a pesar de que sea un trabajo que deba ser en equipo y que es otro campo distinto, parece que nosotros siempre estamos por debajo y eso se ve todavía. O sea habrá médicos que no, pero la inmensa mayoría desde la universidad pues eso, lo maman, que son los dioses del mundo por resolver la enfermedad y bueno pues la enfermera siempre aparece por debajo. (GD1)

Pero es que de todas maneras se complica más la cosa, porque no se trata sólo de ganas de cambiar las cosas con respecto a los compañeros y tal, sino con respecto a los pacientes, porque hay muchos pacientes que todavía tienen ese ideal de la enfermera de antes, que era la practicante o la secretaria del médico, entonces el tener que cambiar esa concepción que tiene todavía mucha gente de que ;ah! ¿pero esto me vas a atender tú? o ¿esto no me lo debía, no se lo deberías de comentar al médico? (GD1)

- Y te preguntan si eso es una carrera universitaria.

- Pero lo que es amigos míos, de mi edad, universitarios, que te digan eso pues te quedas... me derrumba, te deprime un poco, pero eso así en general... (GD1)

- Pero, ya sólo por el hecho de que te llamen de usted. A mí me da exactamente igual, yo para todo el mundo soy A... y soy A..., pero el hecho de que en un sitio te hablen de usted, te hablen con respeto, al hecho de que en otro sitio te digan, shss...

- Camarero, como me han llamado a mí.

- Guapa vente para acá, o camillero. (GD2)

Yo a lo que voy, o a lo que iba, que me parecía muy bien mi trabajo, lo que has dicho tú antes, pero que, en general a la población, pero desde los niños pequeños hasta el mayor más mayor, ¿me entiendes?, darnos a conocer, porque realmente en primaria ¿quién nos conoce? (GD2)

- Porque hay médicos que la primera vez que te ven yo no sé lo que piensan que debe ser un enfermero, lo que ha estudiado y entonces te tratan como si fueras tonto, entonces, claro dices, yo no sé lo que este tío lo que se está pensando que soy yo.

- Por eso también será que los médicos no tienen buena confianza en nosotros, porque siempre nos han visto como un cuerpo técnico que iba a hacer lo que ellos querian que hicieras.

- Es que como no nos valoran, pues... (GD3)

Nos conocen... en el hospital, saben que existimos, pero no saben lo que hacemos, saben que hay enfermeras, pero no saben exactamente decirte quién es ni qué es lo que hace la enfermera. (GD3)

Nosotros estamos moviéndonos, anónimamente, y nos estamos moviendo mucho, mucho, pero anónimamente, y es lo que contra lo que quizás si que habría que protestar de aqui a poco, de aquí a poco. Porque a poco que hagas un estudio verás que cualquier referencia de los 10 años últimos, por poner una fecha, de los 10 años últimos hacia acá, ningún folleto, ninguna noticia de nuestra propia institución hace referencia a enfermería como actor, agente de salud, ninguna, siempre son de médicos y especializados. (GD4)

Si un paciente necesita de enfermería, primero tiene que tener la conciencia de que necesita ser visto por un enfermero, cosa que no existe actualmente, y cuando eso ocurriera, que ya es mucho ocurrir, tendría que tener la información para citarse conmigo, la información es mi nombre en su tarjeta sanitaria. (GD4)

hospital. Piensan que sus prácticas mejorarían si cuando llegaran a los servicios fueran presentados y se les explicara dónde se encuentra cada cosa, es decir si se les permitiera ser identificados y reconocidos.

En el caso de los tutores de atención especializada la insatisfacción está producida tanto por el desconocimiento de los objetivos docentes, como por la falta de formación que declaran tener para desarrollar esta función (falta de reconocimiento a su labor como docentes). Incluyendo a los participantes en el grupo de exalumnos que ya trabajan en el hospital, viven la docencia como una imposición, piensan que no están preparados para ser docentes (no está reconocida por parte de las instituciones la necesidad de formarlos para que a su vez ellos puedan formar) y que los alumnos llegan sin que nadie se los presente ni les explique los objetivos. Sobre el nivel de conocimientos de los alumnos, los tutores de especializada, incluyendo a los exalumnos, piensan que los estudiantes llegan mal preparados para el trabajo del hospital y poco motivados para aprender a realizar técnicas, debido a que la Escuela prima el aprendizaje de la enfermería comunitaria. Esto se puede interpretar como la falta de reco- 
nocimiento a una de las labores principales de la enfermería. Los tutores de atención primaria señalan que la preparación de sus actividades docentes la hacen a costa de su tiempo libre y que no han recibido formación para dar docencia, salvo la que se proporcionan y financian ellos mismos, a pesar de que debería ser responsabilidad de la Universidad. Su insatisfacción procede también de que la labor como tutor se vive como impuesta, no está remunerada, es decir, no está valorada, ni es reconocida como mérito en sus curriculum. Opinan que no existe una verdadera evaluación de la tutoría (es decir, reconocimiento de la misma). También en este grupo piensan que los alumnos precisan más formación, tanto en en enfermería comunitaria como en el resto de las materias básicas.
Los tutores del hospital piensan que los alumnos pasan demasiado tiempo de prácticas en atención primaria y que hacen pocas de hospital. Los tutores de atención primaria opinan que los estudiantes pasan mucho tiempo en el hospital y demasiado poco en atención primaria, lo que en ambos casos puede traducirse como falta de reconocimiento a su profesión por parte de unos y otros (tabla 8).

Lo que más llama la atención del discurso en los grupos es que el conjunto del personal de enfermería, sean alumnos o profesionales, trabajando en la atención primaria o en la especializada, se expresa a través de la queja, que se manifiesta con los significantes angustia, ansiedad y estrés desde que empiezan a estudiar y continúa después de

Tabla 8

Sobre las prácticas durante la diplomatura

No, porque yo por ejemplo considero que en mí el seguimiento que a mi se me hizo en mis prácticas el año pasado [en atención primaria] fue insuficiente. Porque yo con la persona con la que estuve, vale, era tutora voluntaria pero no se ocupaba de tutorizar al alumno, yo no lo vi. (GD1)

No, y en especializada cosas... también es que muchas veces, lo que decimos, que dicen que tú vas a ser el tutor, y a lo mejor esa persona no está preparada o no quiere. Yo me he encontrado de llegar a unas prácticas y decir: ah, es que eres mi alumno, y no me importa que tú vayas detrás de mi mirando lo que estoy haciendo, pero a mí no me gusta enseñar y no es sólo que no me guste es que no sé, entonces como no sé explicar lo que estoy haciendo, pues coge lo que puedas. Entonces eso, en un primer contacto con un hospital, pues eso... (GD1)

A mí tampoco, a mi me han asignado una alumna, pero además como tal, durante todo su rotatorio de prácticas, yo soy su tutora y tengo que hacer su evaluación y demás, y a mi nadie me ha consultado... (GD2)

Es lo que siempre hemos criticado cuando hemos hecho las prácticas, que el tutor o la persona que está contigo no conoce tus objetivos, lo cual hace pie a que no aprendes lo suficiente, no te encamina, toda esa historia que todos conocemos perfectamente. Y ahora nos vemos desde el otro lado y dices es que bueno, en realidad el problema es que de por sí están mal organizadas las prácticas, o sea partiendo de esa base, tanto por un sitio como por otro. (GD2)

- Y encima te está diciendo que un trabajo que tú estás haciendo cada día [refiriéndose a las técnicas] no es importante, también es... y dices bueno, pues nada, no será importante. Y que luego la mayoría [de los estudiantes de enfermería] cuando acaban es que trabajan en hospitales, porque en primaria hay menos..

- Pero aparte porque ellos mismos dicen que en la Escuela dicen que eso no es importante, que eso ya se aprenderá. (GD3)

Alli ni objetivos, ni qué tiene que hacer, ni qué tiene que deshacer, alli llegan y [...] dicen que ésta es una alumna que ha venido y se acabó y luego ya te enterarás tú si era de primero, de tercero, de segundo. (GD3)

Pero si resulta que estás dedicando un tiempo que no ves ningún fruto de ningún tipo, ni de mejora económica, ni de mejora a nivel de formación, de currículos, ni de nada, dices pero bueno ja qué estamos jugando? No, a mí me parece que ése es un tema desde luego, para los tutores muy importante y en la docencia conforme se está llevando hoy en día. (GD4)

Te acaba desmotivando, porque claro, no nos reconocen las horas que hacemos de prácticas, es decir, las horas que dedicamos a los alumnos, no nos las reconocen de ninguna manera. Preguntas qué se puede hacer con eso, y te dicen, bueno te damos un papel, pero que eso ni vale para oposición, ni vale absolutamente para nada. Si no te pagan, si no te revalorizan tu tiempo que tienes que dedicar a esas cosas, llega un momento que dices, mira... (GD4) 
llevar años trabajando. Esa angustia parece estar ocasionada por la falta de reconocimiento social que padecen y que les impide estar dotados de identidad como colectivo. En todos los grupos se critica al personal de enfermería que no son ellos mismos, no hay una defensa de su profesión, ni de los sujetos que la desarrollan. Y aunque los factores que señalan podrían justificar por sí solos la aparición de la queja, que sin embargo no provoca ningún tipo de movilización colectiva para buscar soluciones, lo que aparece por encima de ella es la angustia que les bloquea. Al no identificarse como colectivo carecen de objetivos comunes y de la suficiente cohesión para organizar sus reivindicaciones, lo que les convierte en personas muy pasivas respecto a las mismas. Cuando se quejan de tener que resolver funciones que parecen no ser responsabilidad de nadie en concreto, se están quejando de ser un cajón de sastre donde acaba todo lo que no está identificado. Cuando se quejan de ser trasladados de un servicio a otro, se están quejando de ser utilizados de comodín que para todo vale, de piezas a las que no se le reconoce un valor propio en el servicio en el que estaban ni se les va a reconocer en el servicio al que van. Cuando se quejan de la falta de trabajo en equipo en la asistencia hospitalaria se puede seguir entendiendo la queja en el mismo sentido; donde un conjunto de individuos no se identifica como colectivo no puede haber un equipo de ellos y se vuelven hacia los enfermeros de atención primaria que sí trabajan de esta forma. De hecho estos profesionales de atención primaria, son los más satisfechos (trabajan en equipo, luego existen, tienen las funciones definidas y su labor es reconocida por los médicos), pero también a ellos les sigue faltando el reconocimiento de la población a su labor y por eso se quejan de que la misma no se evalúe, es decir, no se valore, no se reconozca. A pesar de ello, los profesionales de atención primaria son los únicos que se identifican como colectivo a través de la diferencia con el personal de enfermería del hospital y para institucionalizar esta diferencia reclaman que la enfermería de atención primaria sea una especialidad. Al funcionar como colectivo ya son capaces de plantear reivindicaciones laborales, como la remuneración de las prácticas y que la docencia que imparten se reconozca en sus curriculum. Cuando reclaman la licenciatura hablan además de su contenido, que ha de incluir formación en materias como la investigación, y del aumento de sueldo que debe conllevar.

La falta de identificación de los profesionales de enfermería como colectivo que se detecta en el discurso de todos los grupos, basada en la falta de reconocimiento, social, justificaría la insistencia en pedir la licenciatura y las especialidades, lo que contribuirían a su reconocimiento, primero por parte de las instituciones y como consecuencia por parte del resto de la sociedad, lo que podría resolver su angustia. Mientras la angustia no desaparezca en los profesionales de enfermería, estos no dejarán de transmitirla a sus alumnos, lo que explica que la misma esté ya presente desde el comienzo de los estudios. Y si la angustia es el mediador de las relaciones que se dan entre los alumnos que realizan sus prácticas y los profesionales de enfermería que las imparten, difícilmente ninguno de los dos colectivos podrá estar satisfechos con ellas.

\section{DISCUSIÓN}

Por los resultados de esta investigación podemos concluir que tanto los alumnos de enfermería como los profesionales se muestran insatisfechos con la realización de las prácticas clínicas durante la diplomatura. Esta insatisfacción, como hemos visto, está relacionada con tres factores: 1) la falta de reconocimiento social hacia su profesión, 2) que les impide identificarse ellos mismos como colectivo y 3) que hace que la angustia, el estrés y la ansiedad sean los principales protagonistas durante su trabajo, que incluye la docencia durante las prácticas. Hay factores que dependen tanto de la Escuela como de las instituciones sanitarias que par- 
ticipan en la docencia que, objetivamente, si fueran mejorados podrían aumentar la satisfacción respecto a las prácticas, pero en ellos subyace el malestar de un conjunto de profesionales que no se reconocen entre sí como colectivo porque no se sienten reconocidos por la sociedad.

En atención primaria, tal como han evidenciado otras investigaciones anteriores ${ }^{17,18}$, el personal de enfermería ha encontrado una oportunidad para su identificación en las diferencia con el personal del hospital. Esto mismo debe conseguirse con los profesionales que prestan sus servicios en el hospital, que es, por otra parte, donde trabajan la mayoría de los profesionales de enfermería, los cuales son los que más angustia declaran en este trabajo, al igual que en otros ${ }^{19}$. Es, pues, necesario dotar a este colectivo de una identidad que en la actualidad no tiene y que justifica su pasividad hasta con sus propios intereses. Reflejo de ello puede encontrarse en el hecho de que fue el grupo de tutores de atención especializada (GD3) el único que planteó problemas a la hora de realizar la reunión para esta investigación, lo que puede interpretarse como un ejemplo más de su falta de implicación en actividades relacionadas con su profesión.

Tanto en atención especializada como en atención primaria no existe una estructura sólida sobre la que se apoye la función docente, por lo que el proceso de aprendizaje se vive, tanto por tutores como por alumnos, como una cuestión que no tiene un valor reconocido por parte de las instituciones. $\mathrm{La}$ licenciatura, el reconocimiento universitario y el reconocimiento profesional y con él el social, les proporcionaría la posibilidad de una carrera docente de la que hoy carecen, ya que al no ser licenciados no pueden tampoco acceder a un doctorado específico en enfermería. En la actualidad tienen que recurrir a otras licenciaturas para poder conseguir el grado de doctor y con ambos, licenciatura y doctorado, aumentar su acceso a las plazas de profesores en las escuelas de enfermería.
Los resultados de esta investigación coinciden con los de otras anteriores en lo relativo a la angustia y estrés que sufre el personal de enfermería del hospital, así como en lo referente a los factores que los condicionan, como la sobrecarga laboral, la rotación por los servicios, etcétera ${ }^{19}$. Sin embargo, en relación a la satisfacción de los alumnos y de los profesionales de enfermería sobre las prácticas que se realizan, los resultados son los contrarios a los de otras investigaciones ${ }^{1,20,21}$, lo que se puede deber a la metodología utilizada, el grupo de discusión, que ha permitido relacionar la falta de reconocimiento social hacia la profesión de enfermería con su falta de identificación como colectivo y la angustia que ello les provoca con la influencia de estos tres factores durante la realización de las practicas de los alumno. En la metodología cualitativa a través de los grupos de discusión, los sujetos del habla son los propios participantes en el grupo, no el investigador. La ausencia de preguntas por parte de éste ofrece a los participantes en los grupos la libertad de interaccionar entre ellos a partir de la intervención inicial, sin estar sujetos a las respuestas cerradas que un cuestionario condiciona, por lo que es una metodología capaz de hallar respuestas nuevas, no recogidas hasta entonces en otras investigaciones ${ }^{22,23}$.

Una posible limitación de este trabajo es el hecho de que los participantes en los grupos se conocían entre ellos, por pertenecer, los alumnos y los exalumnos a la misma escuela de enfermería, lo que implica una serie de relaciones previas al establecimiento del grupo $9,13,15,16$. Otra posible limitación se refiere a la constitución del grupo de tutores de atención especializada (GD3), ya que asistieron sólo tres personas de las convocadas, no dándose con ello las condiciones ideales de realización del grupo, ya que debería haber estado formado por más perso-

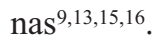

Escuchar tanto la opinión de los alumnos, como ya se apunta en otros trabajos, así como de los propios profesionales de enfer- 
mería ha señalado problemas que aunque aparentemente no estén relacionados con la docencia influyen en ella ${ }^{4}$.

Como conclusión, sentirse sujetos en la relación con los médicos y menos dependiente de ellos; en la relación con los pacientes y en la que deberían establecer con la escuela para formarse como docentes, para conocer los objetivos con los alumnos y para poder informar de la realidad de la actividad asistencial, son temas pendientes en el desarrollo de la profesión de enfermería. La definición de las funciones, la protocolización de las actividades y la realización de evaluaciones del proceso asistencial de enfermería, como principio de su formación continuada, redundaría en una mejor prestación de servicios y una mejor función docente. La mejora de las condiciones laborales en el hospital, además de disminuir su estrés, facilitará el establecimientos de los vínculos necesarios, tanto entre los profesionales de enfermería como con el trabajo que realizan, motivándoles para su formación continuada y para la realización de trabajos de investigación. La evaluación del trabajo de enfermería, tanto en atención primaria como en especializada, identificándoles como responsables de los resultados obtenidos, el establecimiento de la licenciatura junto con el desarrollo de diferentes especialidades, así como la promoción de la investigación sobre su trabajo y el conocimiento de sus resultados por parte de la sociedad, contribuirán asímismo al reconocimiento social de los profesionales de enfermería y por lo tanto a su identificación subjetiva como colectivo. Con ello se permitirá el desarrollo de una carrera asistencial, docente e investigadora propias, que proporcionará una mayor satisfacción profesional y la mejora de la calidad de los cuidados que prestan.

\section{AGRADECIMIENTOS}

A Marián Gil Nebot por su participación en diferentes momentos de la investigación.
A los participantes en los grupos de discusión. A la Escuela Universitaria de Enfermería de la Comunidad de Madrid. A Noé Cuerva Socuéllamos y Mercedes López Santamaría que hicieron las transcripciones.

\section{BIBLIOGRAFÍA}

1. Úbeda I, Pujol G, Olivé C, Álvarez R, Segura MS. Opinión de las enfermeras sobre las experiencias clínicas docentes. Enferm Clín 1993: 3 (1): 11-15.

2. Colina J, Medina JL. Construir el conocimiento de enfermería mediante la práctica reflexiva. Revis ROL Enferm 1997: (232): 23-30.

3. Mompart MP Educación y Salud: Comunicando. XIV Sesiones de Trabajo de la AEED: Cuidados y comunicación. 145-163. Burgos, dic. 1993.

4. Bernat R, Bordaji T, López J. La comunicación como elemento facilitador del aprendizaje. XIV Sesiones de Trabajo de la AEED: Cuidados y comunicación. 241-250. Burgos, dic. 1993.

5. De León A. Experiencia de integración teoría-práctica en los estudios de enfermería. Rev ROL Enferm 1985: (88): 27-29.

6. Karolinska Hospital. Clinical nursing education at the Karolinska Hospital for students from the Red Cross College of Nursing and Health. Clin Educ KS. 1996.

7. School of Nursing at the Rigshospital. Plan of the Nursing Education. (Trabajo inédito). Copenhagen, jun. 1993.

8. Albéniz C, Antón MV, Pérez JM, Ramasco M. La escuela Universitaria de la Comunidad de Madrid: un proyecto en marcha. Madrid: Consejería de Sanidad y Servicios Sociales; 1995.

9. Métodos y Técnicas cualitativas de investigación en ciencias sociales. Madrid: Síntesis Psicológica; 1994.

10. Alonso LE. La mirada cualitativa en sociología. Madrid: Fundamentos; 1998.

11. El análisis de la realidad social. Métodos y Técnicas de investigación. Madrid: Alianza Universidad; 1990.

12. Schartz la y Jacobs H. Sociología cualitativa; método para la reconstrucción de la realidad. México: Editorial Trillas. 
13. Canales M, Peinado A. Grupos de discusión. En: Métodos y técnicas de investigación en ciencias sociales. Madrid: Síntesis psicológica; 1994. p. 287-316.

14. Murillo de la Vega S. Introducción a las técnicas cualitativas en un marco sanitario. En. Enfermería comunitaria 2. Epidemiología y enfermería. Sánchez Moreno ed. Madrid: McGraw-Hill Interamericana; 2000. p. 137-47.

15. Ibáñez J. Cómo se realiza una investigación mediante grupos de discusión. En: El análisis de la realidad social. Métodos y Técnicas de investigación. Madrid: Alianza Universidad; 1990. p. 489-501.

16. Alonso LE. Entre el pragmatismo y el pansemiologismo. En: La mirada cualitativa en sociología. Madrid: Fundamentos; 1998. p. 57.

17. Pérez Andrés c. Aceptación del contrato programa por el personal sanitario de la atención primaria de Madrid: un estudio cualitativo. Rev Sanid Hig Pública 1995; 69:79-88.
18. Corrales D, Galindo A, Escobar MA, Palomo L y Magariño MJ. El debate sobre la organización, las funciones y la eficiencia de enfermería en atención primaria: a propósito de un estudio cualitativo. Aten Primaria 2000; 25: 214-219.

19. Fornás Vives J. Respuesta emocional al estrés laboral. Rev Enferm 1994; 31-39.

20. Ramasco M. Calidad de la enseñanza en la Escuela Universitaria de Enfermería de la Comunidad de Madrid. (Documento mimeografiado). Madrid; 1995.

21. Zapico Yáñez F, Blanco Sánchez R, Consul Giribert M y Juanola Pagés M. ${ }^{\mathrm{a}} \mathrm{D}$. Aprendizaje y satisfacción de los estudiantes de enfermería en las prácticas clínicas (I). Hospital. Enfermer Clín; 7: 20-28.

22. Alonso LE. El grupo de discusión en su práctica. En: La mirada cualitativa en sociología. Madrid: Fundamentos; 1998. p. 105.

23. Pérez Andrés C. ¿Deben estar las técnicas de consenso incluidas entre las técnicas de investigación cualitativa? Rev Esp Salud Pública 2000; 319-21. 